\title{
Correlations between Biomechanical Characteristics, Physical Characteristics, and the Ability to Maintain Dynamic Sitting Balance on an Unstable Surface in the Disabled with Spinal Cord Injury
}

\section{Solbi Kim, Yoonhee Chang, Gyoosuk Kim}

Korea Orthopedics \& Rehabilitation Engineering Center (KOREC); Research and Development team, Incheon, 403-120

\section{Corresponding Author \\ Yoonhee Chang \\ KOREC, R\&D team 9-15 Gusan-dong, \\ Bupyeong-gu, Incheon, 403-120 \\ Phone : +82-32-500-0773 \\ Email : yhchang@korec.re.kr}

Received : November 18, 2013

Revised : January 17, 2014

Accepted : January 27, 2014
Copyright@2014 by Ergonomics Society of Korea. All right reserved.

Co This is an open-access article distributed under the terms of the Creative Commons Attribution Non-Commercial License (http:// creativecommons.org/licenses/by-nc/3.0/), which permits unrestricted non-commercial use, distribution, and reproduction in any medium, provided the original work is properly cited.
Objective: This study aims to analyze the factors that affect the ability to maintain dynamic sitting balance (DSB), biomechanical characteristics, and physical characteristics in spinal cord injuries (SCI) patients.

Background: Virtual ski training systems, ski equipment, and training protocols for disabled skiers are being studied to spread awareness. However, few studies have been reported on the sitting balance ability associated with chair mono skiing.

Method: A dynamic sitting balance border system was built to investigate the ability to maintain dynamic sitting balance in SCI patients. Trunk muscle activity was evaluated by electromyogram while conducting dynamic sitting balance tests. The trunk muscle strength was tested with a portable handheld dynamometer. Physical activity scores were measured with the physical activity recall assessment.

Results: There were high levels of correlation between the ability to maintain DSB and trunk flexor strength, extensor strength, rotator strength, and physical activity score. However, height, weight, and injury level in SCI patients were not correlated with the ability to maintain DSB. Additionally, strong negative correlations were found between muscle activities of the external oblique and lumbar erector spinae muscles and the ability to perform the backward tilt test. Trunk extensor muscle activity during the ball lifting test was significantly higher than in other tests.

Conclusion: The results indicate that improving trunk muscle strength and physical activity can increase the ability to maintain DSB.

Application: The findings of a close relationship between trunk strength, physical activity, and the ability to maintain DSB need to be reflected in the chair mono ski training program.

Keywords: Chair mono ski, Disabled with spinal cord injury, Sitting balance, Strength, Muscle activity

\section{Introduction}

The population enjoying skiing and snowboarding increases each year, since winter sports become popular. The interests of disabled people and general public in winter sports for disabled people are on the rise with the scheduled hosting of the 2018 Pyeongchang Winter Olympics. Korea's winter sports for disabled people, however 
are not activated, due to the lack of relevant facilities, disabled people's awareness on the sports and information. This can be identified from the lack of athletes with disability. The athletes with qualifications to participate in the international individual winter sports games are 6 in skiing and 2 in snowboarding. Among them, the disabled people actually taking part in international events are only 3 in skiing. Disabled people's skiing is divided into standing, sitting and blind skiing. The sports games are carried out by making fair conditions through awarding additional points according to disability grade (Petrofsky 1997; Uh 2009). Sitting ski sport is more distributed than other ski sports, because it offers safety and pleasure simultaneously (Uh 2009), and Korea's disabled national team athletes also use chair mono skis. Concerning the chair mono skis used by spinal cord injuries (SCI) and spinal cord cut patients, the skiers use outrigger, a supplementary tool, with both hands, moves his/her weight using upper body while sitting on the seat, and controls strength. For this reason, the upper extremity muscle strength and sitting balance are required (Uh 2009).

The disabled with spinal cord injuries show unstable standing posture, due to damaged posture control ability, deriving from the lack of trunk stability and balancing ability, in view of physical factors like injury level and trunk muscle strength (Seelen et al., 1997; Serra-Añó et al., 2012). Since their posture is not stable essentially, their sitting balance becomes very unstable even with a slight movement of the upper body (Klefbeck et al., 1996; Serra-Añó et al., 2012). In sitting skiing, where there are many upper extremity's movements, sitting balance is a very important factor for a disabled person with spinal cord injuries, and thus, trunk stability training is truly essential.

Recently, virtual ski training equipment and protocol for sitting skiing for paraplegia people are researched and developed in order to improve adaptability to skiing with the diffusion of winter sports for disabled people prior to participating in actual chair mono ski sport games (Uh 2009; Hwang 2010; Chang et al., 2013). The study of Pernot (2011) is the only study on sitting balance in relation with sitting skiing, and he reported static sitting balance and body center. However, sitting skiing is closely associated with dynamic balance using upper extremity and trunk, but such a study is still insufficient.

The purpose of this study is to identify correlations between factors by assessing dynamic sitting balance (DSB), trunk muscle strength, physical characteristics and trunk muscle activity targeting the disabled with spinal cord injuries (SCI). The findings can be used as basic data for training equipment and relevant apparatus development, as well as protocol establishment on sitting skiing training for disabled people.

\section{Method}

\subsection{Participants}

This study targeted 8 people with paraplegia, who were diagnosed as SCI patients. The participants (subjects) in this study are those who have spinal cord injuries at T9 or below vertebrae, which enable a person to sit on a wheelchair by straightening upper body. Those participants had to have no muscular skeletal diseases and neurologic symptoms. Those who had fracture, lower extremity's weight load contraindicate, skin damage or bedsore, orthostatic hypotension and cardiovascular disease were excluded from the subjects of this study. After offering sufficient explanations on the experiments, this study conducted the experiments.

\subsection{Tasks and apparatus}

\subsubsection{Sitting balance assessment}

This study manufactured a jig by installing a chair on the hemisphere to measure sitting balance on the unstable board (Figure 1). 
For smooth backward movement, the back of the chair was removed. To prevent a falling accident, the stability guide cushion bar was installed in an effort to cope with a possible danger.

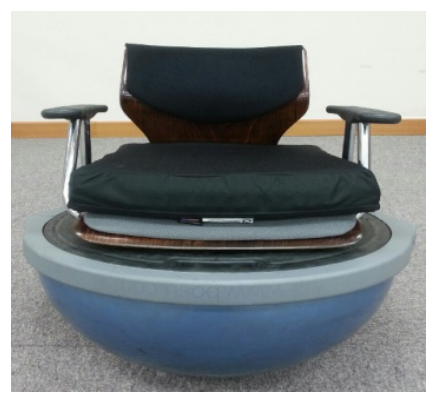

Figure 1. The actual figure of the test-balance board which developed from KOREC

Sitting balance ability test was evaluated by revising/modifying the test-table-test, the sit ski disability grade standard test (Pernot et al., 2011). Existing tests were measured on the stable surface, and $45^{\circ}$ forward and backward tilt was possible, but the risk of an accident is huge on the unstable surface; therefore, the standard angles were revised to be forward $30^{\circ}$ and backward $10^{\circ}$ (Table 1). All the subjects' forward and backward maximum tilting angles were measured on the static surface, when they sat on the experiment table prior to the experiment. The standard angle was set with approximate value of the measured mean angle. To test sitting balance, the subjects sat on the jig (modified balance board), which was manufactured with placing both hands on the sides of the trunk, straightening upper bodies. A tester fixes subject's knees using strap, and place the subject's knees to be bent by $90^{\circ}$. And then, the tester held subject's ankle with tester's hand and tested four functions of balance, after fixing the location of the ankle using tester's weight.

Table 1. Grading of the functional assessment on the test-balance board (Modification based on Pernot's study)

\begin{tabular}{c|l|l|l|l}
\hline Score & \multicolumn{1}{|c|}{ Test 1 } & \multicolumn{1}{c|}{ Test 2 } & \multicolumn{1}{c}{ Test 3 } & \multicolumn{1}{c}{ Test 4 } \\
\hline 0 & $\begin{array}{l}\text { The subject cannot lean } \\
\text { forward, loses balance. }\end{array}$ & $\begin{array}{l}\text { The subject cannot lean } \\
\text { backward, loses balance }\end{array}$ & $\begin{array}{l}\text { The subject cannot sit with } \\
\text { the arms abducted. }\end{array}$ & $\begin{array}{l}\text { The subject cannot lift the } \\
\text { medicine ball. }\end{array}$ \\
\hline 1 & $\begin{array}{l}\text { The subject can lean some } \\
\text { degrees out of center of } \\
\text { gravity, but lose balance } \\
\text { before } 30^{\circ} .\end{array}$ & $\begin{array}{l}\text { The subject can lean some } \\
\text { degrees out of center of } \\
\text { gravity. He compensates with } \\
\text { the head and increases his } \\
\text { kyphosis position of the } \\
\text { upper spine. }\end{array}$ & $\begin{array}{l}\text { The subject only uses the } \\
\text { arms when trying to rotate. }\end{array}$ & $\begin{array}{l}\text { The subject can lift the } \\
\text { medicine ball, but cannot } \\
\text { hold it with both hands, nor } \\
\text { lift it over the head. }\end{array}$ \\
\hline 2 & $\begin{array}{l}\text { The subject can lean forward } \\
\text { and come up with using the } \\
\text { head and upper part of the } \\
\text { trunk from 30. }\end{array}$ & $\begin{array}{l}\text { The subject can lean } \\
\text { backwards to 10, but cannot } \\
\text { maintain this position. }\end{array}$ & $\begin{array}{l}\text { The subject rotates the upper } \\
\text { body, but one side is better } \\
\text { than the other, or lumbar } \\
\text { spine is not following in the } \\
\text { rotation. }\end{array}$ & $\begin{array}{l}\text { The athlete leans on the } \\
\text { medicine ball when putting } \\
\text { it down. }\end{array}$ \\
\hline 3 & $\begin{array}{l}\text { The subject straightens up } \\
\text { normal and can lean forward } \\
\text { up to } 30^{\circ}\end{array}$ & $\begin{array}{l}\text { The subject straightens up } \\
\text { normal and can lean } \\
\text { backward up to 10. }\end{array}$ & Normal trunk rotation. & Normal function. \\
\hline
\end{tabular}

- Test 1 (forward tilt): A subject leans upper body forward maximum on the jig, and maintains the posture for 5 seconds. 
- Test 2 (Backward tilt): A subject leans upper body backward maximum on the jig, and maintains the posture for 5 seconds.

- Test 3 (Trunk rotation): A subject straightens upper body maximum on the jig, and rotates both arms $90^{\circ}$. The subject slowly rotates trunk left and right once, respectively.

- Test 4 (Ball lifting and moving): A subject straightens upper body maximum on the jig, and lifts a $1 \mathrm{~kg}$ medicine ball over head with both hands. The subject rotates the ball lifted over head left and right, and forward and backward.

The assessment standards of the four tests of balance functions are shown in Table 1, and each function test was measured three times repeatedly. During the sitting balance tests, video was shot at the side to assess the trunk tilting angle of the subjects (a57, Sony, Japan).

The function performance was assessed by defining the angles between the acromion and femoral head, and between the femoral head and femur as trunk tilt angles through the attachment of reflection makers $(20 \mathrm{~mm})$ on the acromion, femoral head and femur.

For the performance score of each balance test, repeatedly measured mean value was used. The total of the four balance function test performance scores was used as sitting balance ability score (Pernot et al., 2011).

\subsubsection{Electromyography}

During the four balances function tests, this study measured muscle activity using a surface electromyography system (Trigno Wireless EMG System, DELSYS., USA) to check trunk muscle use pattern. This study analyzed the data using EMG Works 4.0 Analysis Software (Delsys Inc., USA). Of the muscles involved in trunk stability, this study conducted experiments by choosing four superficial muscles (upper rectus abdominal, externals oblique muscle, erector spinae of thoracic vertebrae part and erector spinae of lumbar vertebrae part) that can be measured by surface electromyography (Anders et al., 2008). As for electrodes attachment location, they were attached to muscle belly using the method presented by the SENIAM project (Hermens et al. 1999). The sample extraction rate of the electromyography signal was set to be $2,000 \mathrm{~Hz}$, and $20 \sim 500 \mathrm{~Hz}$ band pass filter was used. The electromyography signal of each muscle was RMS processed. As for quantification method, this study used muscle activity value measured on the basis of the maximal voluntary contraction (\%MVC by making it percentage).

\subsubsection{Strength of trunk muscles}

After balance function test for all the subjects, the muscle strength for trunk flexion, muscle strength for extension and muscle strength for trunk rotation were measured using the Power Track II Commander, JTECH Medical, UT, and the measuring was conducted randomly. The recess time between each test was 5 minutes, and each test was repeatedly conducted by measuring three times, respectively. For the muscle value of subjects, the mean value of three times repeated measurement was use (Cynn et al., 2006; Kim et al., 2012).

\subsubsection{Physical activity measurement - Leisure time physical activity}

Body activity level was calculated by leisure time physical activity (LTPA) of the physical activity recall assessment for SCI patents. The items were divided into three: (1) Light activity (2) intermediate activity (3) vehement activity. By calculating each day's activity time, activities during the whole week were investigated. This study used only intermediate activity and vehement activity of LTPA items as shown in the preceding studies. The subjects' body activity scores were calculated by dividing total leisure activity time by the number of days (Martin Ginis et al., 2010). 


\subsection{Statistical analysis}

Table 3 shows the independent and dependent variables in this study, and an analysis was conducted using a statistical program, SPSS ver. 20 (SPSS Inc., USA). To check normal distribution, daily sample kolmogroov Smirnov test method was used, and normal distribution was demonstrated only in the muscle activity results. To identify the correlations of subjects' sitting balance ability, trunk muscle strength, body activity level and general characteristics, and correlations between the performance of each balance function test and measured muscle activity, a Spearman correlation analysis (Spearman's rank-correlation coefficient) was conducted. For performance difference between muscle strength of each muscle and each balance function test performance was analyzed through the Friedman test. For muscle use pattern on each balance function test, One-Way ANOVA was conducted. For activity difference between balance tests of each muscle, the repeatedly measured ANOVA was carried out. For post test on significance, the Bonferroni test was carried out, and statistical significance level was set to be $p<.05$.

\section{Results}

Table 2 shows the general characteristics of the study subjects: the abdominal muscle strength was stronger on average, although no statistical significance was shown, with muscle strength for trunk flexion at $0.72 \pm 0.20 \mathrm{~N} /(\mathrm{kg} \times \mathrm{m})$, muscle strength for trunk extension at $0.59 \pm 0.27 \mathrm{~N} /(\mathrm{kg} \times \mathrm{m})$, left muscle strength for trunk rotation at $0.63 \pm 0.18 \mathrm{~N} /(\mathrm{kg} \times \mathrm{m})$, and right muscle strength for trunk rotation at $.64 \pm 0.23 \mathrm{~N} /(\mathrm{kg} \mathrm{x} \mathrm{m})$. According to the body activity level questionnaire survey, the subjects were confirmed to spend $45.0 \pm 49.4$ minutes on daily average with intermediate activity.

Table 2. Anthropometric characteristics of participants

\begin{tabular}{c|c|l|l|c|c|c|c|c}
\hline Subject & Age $(\mathrm{yrs})$ & Injury level & (In)complete & Height $(\mathrm{m})$ & Weight $(\mathrm{kg})$ & Spasticity & ASIA & Post-injury time (yrs) \\
\hline A & 48 & Th10 & Complete & 1.65 & 70 & N & A & 9 \\
\hline B & 50 & Th11 & Complete & 1.68 & 61 & Y & A & 12 \\
\hline C & 55 & Th11 & Complete & 1.68 & 61 & N & A & 9 \\
\hline D & 57 & L1 & Incomplete & 1.74 & 63 & N & C & 11 \\
\hline E & 38 & T9 & Complete & 1.72 & 58 & Y & A & 11 \\
\hline F & 37 & T9 & Complete & 1.70 & 42 & Y & A & 11 \\
\hline G & 52 & L3 & Complete & 1.73 & 78 & Y & A & 19 \\
\hline H & 44 & L1-2 & Incomplete & 1.80 & 57 & Y & B & 7
\end{tabular}

Abbreviations: yrs, years; Th, thoracic; N, no; Y, yes; L, lumbar.

Table 3. Independent variables and dependent variables of study

\begin{tabular}{l|l}
\hline \multicolumn{1}{c|}{ Independent variables } & Dependent variable \\
\hline Strength of trunk muscles & \multirow{2}{*}{ Scores of dynamic sitting balance } \\
\cline { 1 - 1 } Leisure time physical activity & \\
\hline Physical characteristics & \\
\hline Activity of trunk muscles & Activity of trunk muscles \\
\hline Four tests of dynamic sitting balance &
\end{tabular}




\subsection{Ability of sitting balance}

Each balance function test's mean performance score was $1.1 \pm 1.1$ (forward tilt: test 1), $1.0 \pm 0.9$ (backward tilt: test 2), 2.3 \pm 0.7 , (trunk rotation: test 3) and 1.6 \pm 1.1 (ball lifting and moving: test 4), respectively. From the Friedman test, the most convenient performance of the subjects was trunk rotation test, and the backward tilt test was the most difficult performance $\left(\chi^{2}=16.4, p\right.$ $=0.001)$.

\subsection{Muscle activity}

As a result of the repeated measurement variance analysis, trunk flexors (upper rectus abdominal and external oblique muscle) were used at similar level $(p>.05)$ in the four balance function tests. However, erector spinae of thoracic vertebrae part $(F=$ 7.35, $p=0.03)$ and erector spinae of lumbar vertebrae part $(F=9.89, p=0.02)$ showed significant differences between the tests. Although, trunk extensor (erector spinae of thoracic vertebrae part and erector spinae of lumbar vertebrae part) worked similarly in tests 1,2 and 3, it was used more in test 4 than in other tests $(p<0.01)$ (Figure 2-A).

From the One-Way ANOVA for each balance function test's muscle activity pattern, the muscle activity of trunk extensor (lumbar vertebrae part) was higher than that of trunk flexor in test $1(F=4.63, p=0.009)$. On the contrary, trunk flexor (external oblique muscle) was more activated than trunk extensor in test $2(F=2.867, p=0.04)$. There were no statistically significant differences between muscles in tests 3 and $4(p>$.05) (Figure 2-B). In addition, in the four functions tests, upper abdominal showed a tendency of low activation than other muscles overall, although no statistical significance was shown (Figure 2-B).

A

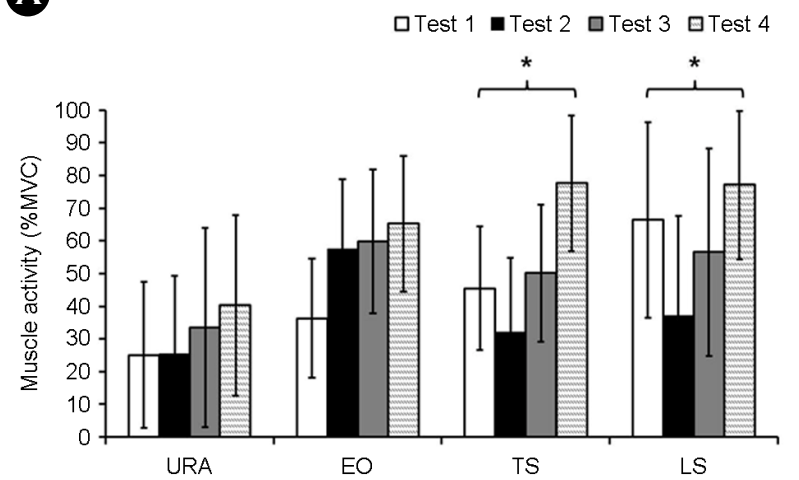

B

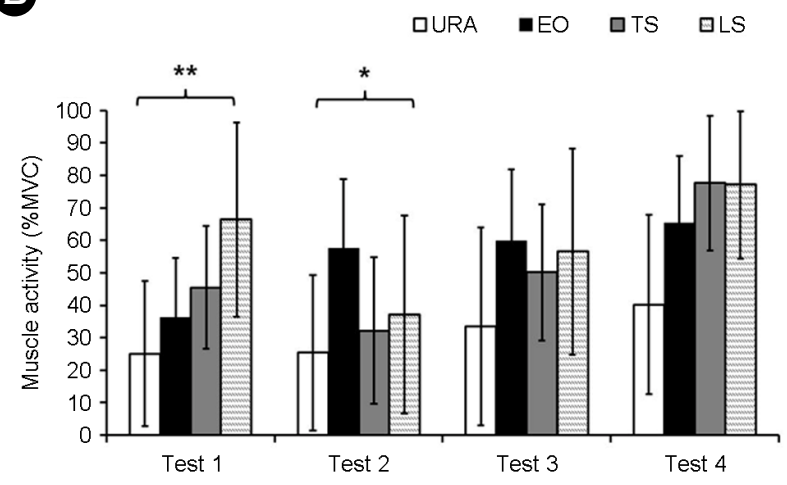

Figure 2. Result of the EMG (\%MVC) of trunk muscles during 4 tests $(A)$ and difference of muscle activity between trunk muscles during tests (B) Abbreviations: URA, upper rectus abdominal; EO, external oblique; TS, thoracic of erector spinae; LS, lumbar of erector spinae ${ }^{\star} p<.05,{ }^{\star \star} p<.01$

\subsection{Correlation between the ability of sitting balance and physical characteristics of participants}

As a result of Spearman Correlation Analysis between the ability to maintain sitting balance and physical characteristics of the participants in this study, the sitting balance has high positive relationships with muscle strength for trunk flexion, muscle strength for trunk extension and muscle strength for trunk rotation, and high correlations were demonstrated in body activity level $(p<0.01)$.

Meanwhile, correlations between spinal injury, height and weight and sitting posture were small, and statistically not significant 
$(p>.05)$ (Table 4).

Table 4. The correlations between the Ability of sitting balance and physical characteristics of participants

\begin{tabular}{l|c}
\multicolumn{1}{c|}{ Physical characteristics } & \multicolumn{1}{c}{ Ability of sitting balance $(r)$} \\
\hline Trunk flexor strength & $.819^{*}$ \\
\hline Trunk extensor strength & $.945^{* *}$ \\
\hline Trunk right rotator strength & $.882^{* *}$ \\
\hline Trunk left rotator strength & $.945^{* *}$ \\
\hline Weight & .317 \\
\hline Height & .000 \\
\hline Leisure time physical activity & $.951^{* *}$ \\
\hline Level & .417 \\
\hline & \multicolumn{2}{|c}{} \\
\end{tabular}

\subsection{Correlation between the ability of sitting balance and activity of trunk muscles}

From the results of Spearman correlation analysis for activity between each balance function performance and muscle strength for trunk muscles, a high negative relationship of muscle activity for external oblique muscle and erector spinae of lumbar vertebrae part was shown only in test 2 (backward tilt) alone $(p<0.05)$ (Table 5).

Table 5. The correlations between the Ability of sitting balance and muscle activity of trunk muscles ( $r$ value)

\begin{tabular}{l|l|l|l|l}
\hline & URA & EO & TS & LS \\
\hline Test 1 & -.655 & -.408 & -.173 & -124 \\
\hline Test 2 & -.504 & $-.819^{*}$ & -.567 & $-.819^{*}$ \\
\hline Test 3 & -.655 & -.408 & -.173 & .124 \\
\hline Test 4 & -.469 & -.505 & -.365 & -.391 \\
\hline
\end{tabular}

Abbreviations: URA, upper rectus abdominal; EO, external oblique; TS, thoracic of erector spinae; LS, lumbar of erector spinae. ${ }^{*} p<.05$

\section{Conclusion}

The aim of this study is to identify the factors affecting balance by examining correlations between the dynamic sitting balance and physical characteristics of disabled people with spinal cord injuries. According to the study findings, there was a high positive correlation between the dynamic sitting balance and trunk muscle strength of disabled people with spinal cord injuries but, no correlation was found with height, weight and spinal cord injury level. Trunk stability is very important in daily life and sports activities. As already identified by numerous studies, trunk muscle enhancement can be connected with athletic activity improvement, if a positive effect is given to functional movement by securing trunk stability more (Kibler et al., 2006; Kim 2010; Granacher et al., 2013). Especially, the trunk stability of disabled people shows instability, unlike general public, and thus, it needs to be essentially evaluated in sports activity. Sitting balance is closely related with trunk stability, and winter sports for disabled people test the ability of sitting balance in consideration of such a relationship (Pernot et al., 2011). The higher spinal 
cord injury level is, the lower trunk muscle strength is clinically. Therefore, awareness that trunk stability is insufficient is strong, as spinal cord injury is higher in general. The findings of this study, however, show that no huge correlation was shown between injury at T9 and below vertebrae and balance. This implies that dynamic sitting balance can be good, if trunk muscle strength is good, despite upper level injury of the spinal cord.

According to the study results, the disabled people leading leisure time with intermediate level and higher had good trunk muscle strength, and dynamic sitting balance was higher. The paraplegic patient's body activity level is widely used for body activity recall evaluation, and is evaluated by dividing it into leisure time body activity, daily life body activity and cumulative body activity. The preceding study that targeted 73 disabled people with spinal cord injuries reported that muscle strength and health had a positive correlation in the intermediate level and higher leisure, but reported that there was no correlation with daily life index (Latimer et al., 2006).

This means that leisure of intermediate level and higher level is more helpful to physical strength and health than spending light daily life. The findings in this study ascertained that those who enjoy leisure have higher dynamic sitting balance and perform more functional movement smoothly, which matches the results of the preceding study.

This study aimed at identifying trunk muscle activity pattern in the case of dynamic sitting balance test using surface electromyography, and using the findings for basic data in future balance training. As a result of electromyography measurement, muscle for extension in the lumbar vertebrae part was used the most with 66.4\%MVC in dynamic sitting balance assessment of forward tilt. External oblique muscle was measured as the highest with $57.5 \% \mathrm{MVC}$ in dynamic sitting balance assessment of backward tilt. In the study of Anders (2006), trunk extensor is activated to maintain posture, when whole body is leaned forward using $3 \mathrm{D}$ spine stabilization training equipment, and it matches the tendency that trunk flexor is used as an agonist. When disabled people maintain forward and backward tilt, the muscles of lumbar vertebrae part (external oblique muscle and erector spinae of lumbar vertebrae part) are mainly used. This is similar to the results of the preceding study asserting trunk central muscle contributes to trunk stability (Cholewicki and McGill 1996; Kibler et al., 2006; Anders et al., 2008; Kim 2010). As for the dynamic training test of backward tilt, the result of this study confirmed high negative correlation between performing ability and lumbar vertebrae part muscles. This implies that posture can be maintained with the use of small amount of muscles, if dynamic sitting balance improves with ensured trunk stability. Such a dynamic sitting balance test can be used as a balance training method in clinical trials. From this perspective, the dynamic sitting balance training of forward and backward tilt is presented as a training method to train lumbar vertebrae part muscle strength of disabled people with spinal cord injuries.

As a result of identifying the activity between dynamic sitting balance tests by each trunk muscle, there was no difference in activity for trunk flexor, but, trunk extensor was greatly activated in the test of balance with upper extremity movement, compared to other tests (77\%MVC on average), which was also statistically significant. This means that trunk extensor's activity increases, compared to other dynamic balance movements, to maintain trunk stability, as the movement of upper extremity becomes severe. There are lots of upper extremity's movements, since weight movement and strength are controlled using upper extremity and balance, and they induce rotation using arms and shoulder muscles in the sitting skiing (Uh 2009). In consideration of such characteristics of skies for disabled people, training on the trunk extensor is considered to be carried out essentially.

Sports for all provide positive effects to quality of life improvement to disabled people, because they can enhance the opportunity for social exchange and harmony with the general public and self-efficacy, in addition to offering physical and spiritual stability (Lee 2004). In this context, research on rehabilitation training through sports is actively conducted, and many technologies and equipment related with sports for disabled people are developed. Although, interests in winter sports for disabled people are on the rise with scheduled hosting of Pyeongchang Winter Olympic Games, the winter sports for disabled people are more difficult to be accessed, compared to other sports for disabled people. Thus, only small number of disabled people participates 
in the winter sports (Burkett 2012; Gastaldi et al., 2012), Also, studies related with such a problem are insufficient. This study identified the factors affecting the dynamic sitting balance of disabled people, and analyzed trunk muscle activity pattern according to dynamic sitting balance test method through a biomechanical analysis. By checking the sitting balance and physical characteristics of the disabled with spinal cord injuries, the guidelines that can be safely enjoyed for sitting skiing can be presented, and the relevant sports injuries can be prevented. While maintaining dynamic sitting balance, the analysis on the trunk muscle activity pattern is expected to be used as basic data in developing sitting skiing equipment and training equipment for disabled people later.

This study was carried out targeting the small number of disabled males with spinal cord injuries, and thus, there is a limitation that it is difficult to generalize the study findings for paraplegic patients. Further study on the dynamic sitting balance and trunk muscle activity change should be conducted, according to gender, age and injury level.

\section{Acknowledgment}

This research was supported by the sports industry technology development project through the Korea Sports Promotion Foundation funded by Ministry of Culture, Sports and Tourism.

\section{References}

Anders, C., Brose, G., Hofmann, G.O. and Scholle, H.-C., Evaluation of the EMG-force relationship of trunk muscles during whole body tilt, Journal of biomechanics, 41(2), 333-339, 2008.

Burkett, B., Paralympic Sports Medicine-Current Evidence in Winter Sport: Considerations in the Development of Equipment Standards for Paralympic Athletes, Clinical Journal of Sport Medicine, 22(1), 46-50, 2012.

Chang, Y.H., Kim, G.S., Ryu, J.C., Kim, S.K. and Mun, M.S. "Characteristics of COP pathway during virtual training on the chair monoski simulator for disabled skier.", Journal of the Korean Society of Precision Engineering. Jeju. 5, 1123-1124, 2013.

Cholewicki, J. and McGill, S., Mechanical stability of the in vivo lumbar spine: implications for injury and chronic low back pain, Clinical Biomechanics, 11(1), 1-15, 1996.

Cynn, H.-S., Oh, J.-S., Kwon, O.-Y. and Yi, C.-H., Effects of lumbar stabilization using a pressure biofeedback unit on muscle activity and lateral pelvic tilt during hip abduction in sidelying, Archives of physical medicine and rehabilitation, 87(11), 1454-1458, 2006.

Gastaldi, L., Pastorelli, S. and Frassinelli, S., A Biomechanical Approach to Paralympic Cross-Country Sit-Ski Racing, Clinical Journal of Sport Medicine, 22(1), 58-64, 2012.

Granacher, U., Gollhofer, A., Hortobágyi, T., Kressig, R.W. and Muehlbauer, T., The importance of trunk muscle strength for balance, functional performance, and fall prevention in seniors: a systematic review, Sports Medicine, 1-15, 2013.

Hermens, H.J., Freriks, B., Merletti, R., Stegeman, D., Blok, J., Rau, G., Disselhorst-Klug, C. and Hägg, G., European recommendations for surface electromyography, Roessingh Research and Development The Netherlands, 1999.

Hwang, S., (The)effects of 12 weeks physical strength training on the alpine mono skiers' aerobic power and anaerobic power. 
Special Physical Education. Yongin, Yongin university: 50, 2010.

Kibler, W.B., Press, J. and Sciascia, A., The role of core stability in athletic function, Sports Medicine, 36(3), 189-198, 2006.

Kim, K.J., Effects of core muscle strengthening training on flexibility, muscular strength and driver shot performance in female professional golfers, Int J App/ Sports Sci, 22(1), 111-127, 2010.

Kim, S.B., Chang, Y.H., Kim, S.K., Bae, T.S., Mun, M.S. and Park, J.C., Changes of Muscle Activation Pattern of Trunk Muscles during Whole-body Tilts with and without Axial Rotation, Journal of the Korean Society of Precision Engineering, 29(7), 805-810, 2012.

Klefbeck, B., Mattsson, E. and Weinberg, J., The effect of trunk support on performance during arm ergometry in patients with cervical cord injuries, Spinal Cord, 34(3), 167-172, 1996.

Latimer, A.E., Ginis, K., Craven, B.C. and Hicks, A.L., The physical activity recall assessment for people with spinal cord injury: validity, Medicine and science in sports and exercise, 38(2), 208, 2006.

Lee, H.-K., The effect of the winter sports participation in self-efficacy of handicapped. Graduate school of leisure science, Korea National Sports University, 2004.

Martin Ginis, K.A., Latimer, A.E., Arbour-Nicitopoulos, K.P., Buchholz, A.C., Bray, S.R., Craven, B.C., Hayes, K.C., Hicks, A.L., McColl, M.A. and Potter, P.J., Leisure time physical activity in a population-based sample of people with spinal cord injury part I: demographic and injury-related correlates, Archives of physical medicine and rehabilitation, 91(5), 722-728, 2010.

Pernot, H., Lannem, A.M., Geers, R.P., Ruijters, E.F., Bloemendal, M. and Seelen, H.A., Validity of the test-table-test for Nordic skiing for classification of paralympic sit-ski sports participants, Spinal Cord, 49(8), 935-941, 2011.

Petrofsky, J., Skiing with a disability, Palaestra Spring, 1997.

Seelen, H.A.M., Potten, Y.J.M., Huson, A., Spaans, F. and Reulen, J.P.H., Impaired balance control in paraplegic subjects, Journal of Electromyography and Kinesiology, 7(2), 149-160, 1997.

Serra-Añó, P., Pellicer-Chenoll, M., Garcia-Massó, X., Brizuela, G., García-Lucerga, C. and González, L., Sitting balance and limits of stability in persons with paraplegia, Spinal Cord, 2012.

Uh, H., Development guidelines of skills training for disabled Mono skier, Korea Institute of Sports Science, 2009.

\section{Author listings}

Solbi Kim: sbkim@korec.re.kr

Highest degree: Master of Science, Department of Ergonomics Therapy, Yonsei University

Position title: Researcher

Areas of interest: Biomechanics, Sports medicine 
Yoonhee Chang: yhchang@korec.re.kr

Highest degree: Doctor of Philosophy, Department of Physical Therapy, Sahmyook University

Position title: Researcher

Areas of interest: Biomechanics, Rehabilitation

Gyoosuk Kim: gskim@korec.re.kr

Highest degree: Doctor of Philosophy, Department of Mechanical Engineering, Yonsei University

Position title: Principal Research Scientist

Areas of interest: Biomimetic, Man Machine Interface and Wearable Robot System 